SUPPORTING INFORMATION

\title{
High-Pressure Neutron Powder Diffraction Study of $\varepsilon$-CL-20: A Gentler Way to Study Energetic Materials
}

Sumit Konar, ${ }^{*}+$ Steven Hunter, $\uparrow$ Carole A. Morrison, $\uparrow$ Paul L. Coster, $\uparrow$ Helen E. Maynard-Casely, $†$ Jonathan G. Richardson, $\uparrow$ William G. Marshall, $\neq$ Annette Kleppe, $\$$ Stewart F. Parker, $\neq$ and Colin R. Pulham* ${ }^{*}$

+EaStCHEM School of Chemistry and Centre for Science at Extreme Conditions, The University of Edinburgh, King's Buildings, David Brewster Road, Edinburgh EH9 3FJ, U.K.

$\neq$ ISIS Neutron and Muon Facility, STFC Rutherford Appleton Laboratory, Harwell, Oxford, Didcot, Oxfordshire OX11 0QX, U.K.

\ Diamond Light Source, Rutherford Appleton Laboratory, Harwell Science and Innovation Campus, Didcot, Oxfordshire, OX110QX, England, U.K.

( ${ }^{*}$ E-mail: sumit.konar@ed.ac.uk; c.r.pulham@ed.ac.uk) 
Table S1: Comparison of the crystallographic parameters for $\varepsilon$-CL-20 calculated at ambient pressure using the DFT-D method alongside results obtained from previous experimental and computational studies.

\begin{tabular}{|c|c|c|c|c|c|c|}
\hline Parameter & Exp. ${ }^{1}$ & Exp & & & ND $^{6}$ \\
\hline$a(\AA)$ & $8.863(1)$ & $8.852(2)$ & 8.9189 & 8.9157 & 8.815 & 8.951 \\
\hline$b(\AA)$ & $12.593(2)$ & $12.556(3)$ & 12.6706 & 12.5171 & 12.526 & 12.284 \\
\hline$c(\AA)$ & $13.395(2)$ & $13.386(3)$ & 13.4251 & 13.4132 & 13.319 & 13.437 \\
\hline$\beta($ deg. $)$ & $106.920(2)$ & $106.82(2)$ & 106.559 & 106.58 & 106.82 & 104.95 \\
\hline$V\left(\AA^{3}\right)$ & $1430.2(3)$ & $1424.2(5)$ & 1454.23 & 1434.68 & 1407.80 & 1427.44 \\
\hline
\end{tabular}

\footnotetext{
${ }^{1}$ Bolotina et al.. J. Appl. Crystallogr. 2004, 37 (5), 808-814.

${ }^{2}$ Nielsen et al. Tetrahedron 1998, 54 (39), 11793-11812.

${ }^{3}$ Konar et al. (this work)

${ }^{4}$ Sorescu et al. J. Phys. Chem. C 2010, 114 (14), 6734-6748.

${ }^{5}$ Tan et al. Phys. B Condens. Matter 2011, 406 (15), 2925-2930.

${ }^{6}$ Bidault et al. RSC Adv. 2019, 9 (68), 39649-39661.
} 
Figure S1 a) Definition of wag angle ( $\delta$ ); b) changes of wag angles with pressure; the values are obtained from DFT calculations

a)

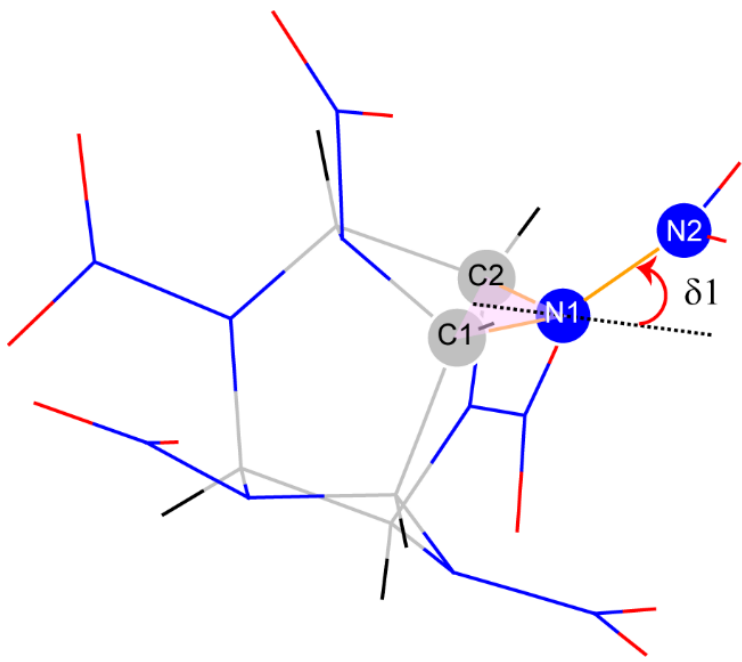

b)

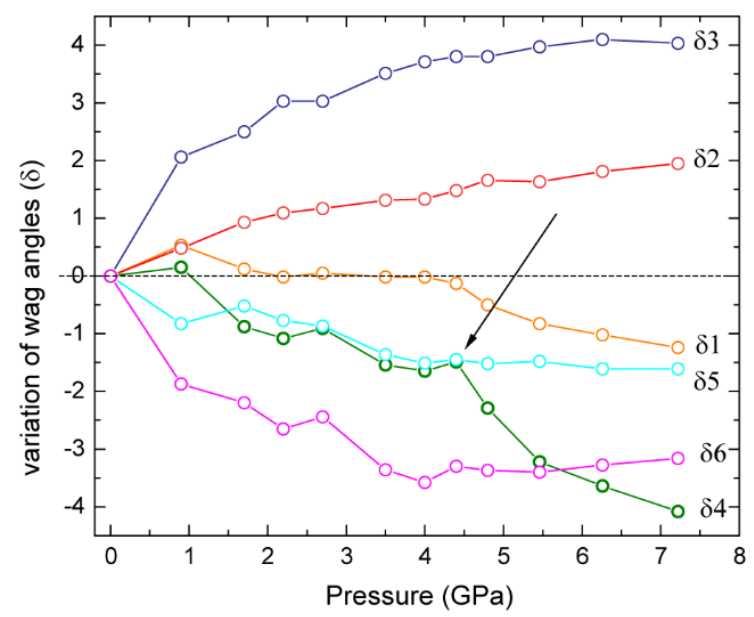

Table S2 Absolute values of six N-NO 2 wag angles of $\varepsilon-C L-20$ at high pressures.

\begin{tabular}{|c|c|c|c|c|c|c|}
\hline & \multicolumn{6}{|c|}{ Wag angle (deg) } \\
\hline $\begin{array}{c}\text { Pressure } \\
(\mathrm{GPa})\end{array}$ & $\delta 1$ & $\delta 2$ & $\delta 3$ & $\delta 4$ & $\delta 5$ & $\delta 6$ \\
\hline ambient & 36.23 & 1.05 & 28.38 & 29.63 & 19.39 & 37.04 \\
\hline 0.9 & 36.76 & 1.53 & 30.44 & 29.78 & 18.56 & 35.17 \\
\hline 1.7 & 36.35 & 1.98 & 30.88 & 28.75 & 18.87 & 34.84 \\
\hline 2.2 & 36.21 & 2.14 & 31.41 & 28.55 & 18.62 & 34.39 \\
\hline 2.7 & 36.28 & 2.22 & 31.41 & 28.72 & 18.52 & 34.6 \\
\hline 3.5 & 36.21 & 2.36 & 31.89 & 28.09 & 18.03 & 33.68 \\
\hline 4 & 36.21 & 2.38 & 32.09 & 27.98 & 17.88 & 33.46 \\
\hline 4.4 & 36.1 & 2.53 & 32.18 & 28.14 & 17.94 & 33.74 \\
\hline 4.8 & 35.73 & 2.71 & 32.18 & 27.34 & 17.87 & 33.67 \\
\hline 5.46 & 35.4 & 2.68 & 32.35 & 26.41 & 17.91 & 33.64 \\
\hline 6.26 & 35.21 & 2.86 & 32.48 & 25.99 & 17.78 & 33.76 \\
\hline 7.22 & 34.99 & 3 & 32.41 & 25.55 & 17.78 & 33.88 \\
\hline
\end{tabular}


TABLE S3 Assignment of the DFT-D computed fundamental eigenvectors computed for $\varepsilon$-CL-20.

\begin{tabular}{|c|c|c|}
\hline Mode no. & $v / \mathrm{cm}^{-1}$ (calculated) & Assignment \\
\hline $1-3$ & 0 & Acoustic \\
\hline $4-22$ & $32.5-70.0$ & Lattice modes \\
\hline $23-79$ & $77.5-173.8$ & Lattice modes + amalgamated $\mathrm{NO}_{2}$ wags/bends \\
\hline $79-100$ & $193.3-259.4$ & Cage deformation bends $+\mathrm{NO}_{2}$ wags/bends \\
\hline $101-112$ & $292.6-308.6$ & Cage deformation str $+\mathrm{N}-\mathrm{N}$ str \\
\hline $113-116$ & $336.5-339.0$ & Cage deformation str \\
\hline $117-128$ & $358.0-375.5$ & Cage deformation bend \\
\hline $129-132$ & $396.9-398.2$ & Cage deformation bend $\mathrm{NO}_{2}$ bend \\
\hline $133-140$ & $439.3-459.5$ & Cage $\mathrm{N}$ oop \\
\hline $141-149$ & $515.9-566.8$ & Cage deformation bend $+\mathrm{NO}_{2}$ bend \\
\hline $150-180$ & $567.8-687.8$ & Cage deformation str and bend and twist $+\mathrm{NO}_{2}$ bends \\
\hline $181-212$ & $701.2-729.9$ & Whole molecule vibration \\
\hline $213-384$ & $764.8-1376.3$ & $\mathrm{~N}-\mathrm{O}$ str \\
\hline $385-408$ & $1636.5-1712.8$ & $\mathrm{C}-\mathrm{H}$ str \\
\hline $409-432$ & $3074.3-3100.9$ &
\end{tabular}


Figure S2 Computed phonon density of states (Brillouin zone $\Gamma$-point) for $\varepsilon$-CL-20
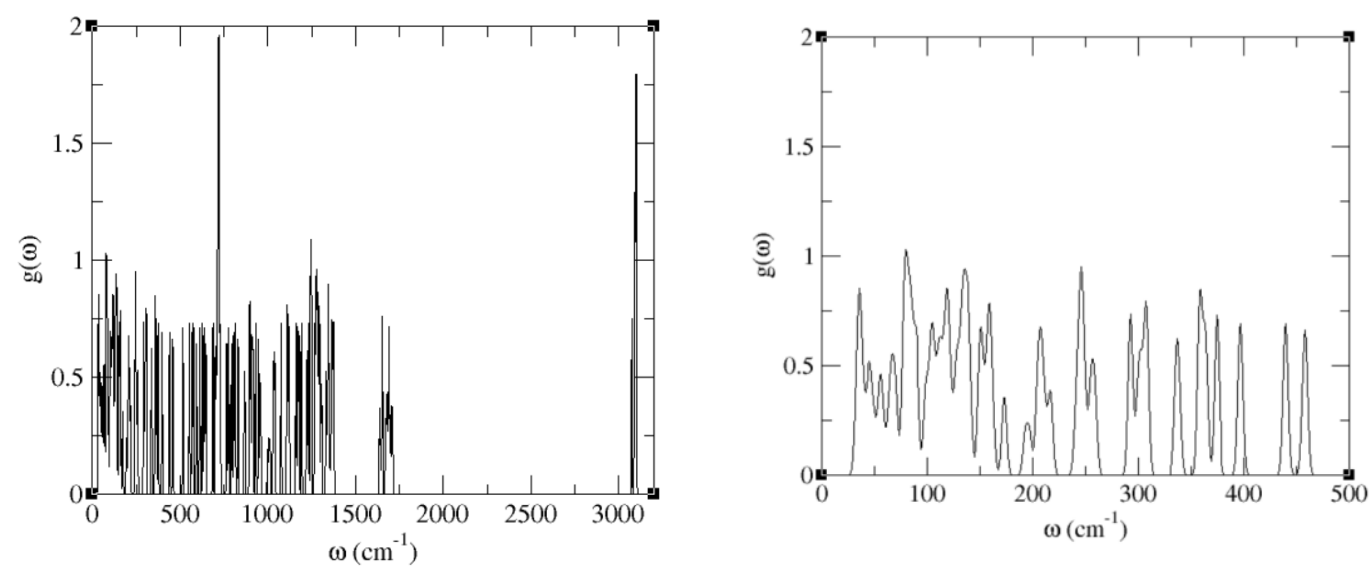
TABLE S4 Comparison of the computationally calculated fundamental modes for $\varepsilon$-CL-20 compared to the INS spectrum reported in this work. * Where there are multiple modes, the DFT-D value stated is an average of the contributing modes. All frequencies, $v$, are in units $\mathbf{~ o f ~}^{\mathbf{c m}^{-1}}$.

\begin{tabular}{|c|c|c|c|c|c|}
\hline Mode* & comp & INS & Mode & comp & INS \\
\hline 4,5 & 33.8 & 28 & $97-100$ & 257.3 & 266 \\
\hline $6,7,8,9$ & 36.7 & 33 & 101-104 & 293.4 & 306 \\
\hline 10 & 41.2 & 40 & $105-108$ & 302 & 314 \\
\hline 11 & 44.2 & 43 & $109-112$ & 307.9 & 318 \\
\hline 12 & 45.3 & 46 & 113,114 & 336.7 & 339 \\
\hline 13 & 47.3 & 50 & 115,116 & 338.7 & 349 \\
\hline 14 & 50.9 & 53 & $117-124$ & 360.9 & 376 \\
\hline 15,16 & 55.5 & 57 & $125-128$ & 374.8 & 386 \\
\hline 17 & 58.1 & 61 & $129-132$ & 397.3 & 412 \\
\hline 18 & 63.4 & 65 & $133-136$ & 440.1 & 450 \\
\hline 19,20 & 66.2 & 69 & $137-140$ & 458.5 & 469 \\
\hline 21,22 & 69.5 & 72 & $141-144$ & 516.7 & 528 \\
\hline 23,24 & 77.6 & 78 & $145-148$ & 553.3 & 572 \\
\hline $25-28$ & 79.8 & 84 & $149-152$ & 567.8 & 584 \\
\hline $29-33$ & 84.8 & 88 & $153-156$ & 575.3 & 595 \\
\hline $34-36$ & 90.3 & 93 & $157-160$ & 593.5 & 607 \\
\hline 37,38 & 99.1 & 103 & $161-164$ & 613.1 & 629 \\
\hline 39,40 & 103.4 & 105 & $165-172$ & 633.2 & 648 \\
\hline $40-42$ & 104.8 & 109 & $173-176$ & 647 & 665 \\
\hline 43,44 & 109.4 & 111 & $177-180$ & 687.2 & 681 \\
\hline 45,46 & 112.7 & 116 & $181-188$ & 706 & 709 \\
\hline 47,48 & 115.7 & 119 & $189-200$ & 717.1 & 727 \\
\hline $49-52$ & 119.4 & 126 & $201-212$ & 724.4 & 753 \\
\hline 53 & 123 & 136 & $213-216$ & 765.9 & 796 \\
\hline $54-56$ & 128.2 & 139 & $217-220$ & 776.6 & 808 \\
\hline $57-59$ & 132.8 & 145 & $221-232$ & 804.1 & 836 \\
\hline $60-62$ & 135.5 & 149 & $233-236$ & 830.4 & 862 \\
\hline $63-64$ & 139.2 & 152 & $237-240$ & 867.7 & 888 \\
\hline $65-66$ & 140.7 & 156 & $257-260$ & 931.2 & 924 \\
\hline $67-70$ & 151.1 & 164 & $241-256$ & 905.1 & 947 \\
\hline 71,72 & 156.4 & 169 & $261-268$ & 952.5 & 991 \\
\hline $73-75$ & 159.4 & 173 & 269,270 & 1001.6 & 1021 \\
\hline 76 & 162.5 & 177 & 271,272 & 1011.1 & 1031 \\
\hline 77 & 172.8 & 183 & $273-280$ & 1035.1 & 1057 \\
\hline 78 & 173.8 & 188 & $281-284$ & 1075.9 & 1095 \\
\hline 79 & 193.3 & 194 & $285-296$ & 1114.8 & 1145 \\
\hline 80 & 197.3 & 199 & $297-312$ & 1175.3 & 1222 \\
\hline $81-84$ & 206.5 & 214 & 313-332 & 1238.3 & 1259 \\
\hline 85,86 & 211.2 & 226 & $333-360$ & 1284.4 & 1310 \\
\hline $87-88$ & 216.8 & 233 & $361-372$ & 1341.2 & 1351 \\
\hline $89-92$ & 244 & 253 & $373-384$ & 1369 & 1391 \\
\hline $93-96$ & 248.2 & 262 & $409-432$ & 3092.2 & 3060 \\
\hline
\end{tabular}


Table S5. Variation of unit cell parameters of $\varepsilon$-CL-20 with pressure, obtained from DFT calculations.

\begin{tabular}{cccccc}
\hline $\begin{array}{c}\text { Pressure. } \\
(\mathrm{GPa})\end{array}$ & $\mathrm{a}(\AA)$ & $\mathrm{b}(\AA)$ & $\mathrm{c}(\AA)$ & $\beta(\mathrm{deg})$ & $\mathrm{V}\left(\AA^{3}\right)$ \\
\hline 0 & 8.9189 & 12.6706 & 13.4251 & 106.5587 & 1454.22 \\
$0.9(2)$ & 8.7538 & 12.3403 & 13.1587 & 105.6479 & 1368.78 \\
$1.7(2)$ & 8.678 & 12.0989 & 13.004 & 104.7637 & 1320.27 \\
$2.2(2)$ & 8.6301 & 12.0148 & 12.9315 & 104.5233 & 1298.01 \\
$2.7(2)$ & 8.593 & 11.9559 & 12.873 & 104.5745 & 1279.98 \\
$3.5(2)$ & 8.5501 & 11.8325 & 12.7629 & 104.2722 & 1251.36 \\
$4.0(2)$ & 8.5216 & 11.7742 & 12.7086 & 104.1699 & 1236.32 \\
$4.4(2)$ & 8.4949 & 11.7397 & 12.6778 & 104.1666 & 1225.88 \\
$4.8(2)$ & 8.4873 & 11.669 & 12.6277 & 103.8418 & 1214.31 \\
$5.46(2)$ & 8.4613 & 11.5961 & 12.5691 & 103.5512 & 1198.92 \\
$6.26(2)$ & 8.4297 & 11.5285 & 12.5076 & 103.4357 & 1182.24 \\
$7.22(2)$ & 8.4018 & 11.4546 & 12.4338 & 103.3928 & 1164.08 \\
\hline
\end{tabular}


Figure S3 Rietveld refinement plots of X-ray powder diffraction patterns at elevated pressures $[\lambda=0.4847 \AA$ (up to $7.2 \mathrm{GPa})$ and $\lambda=0.3727 \AA$ ( above $7.2 \mathrm{GPa})]$
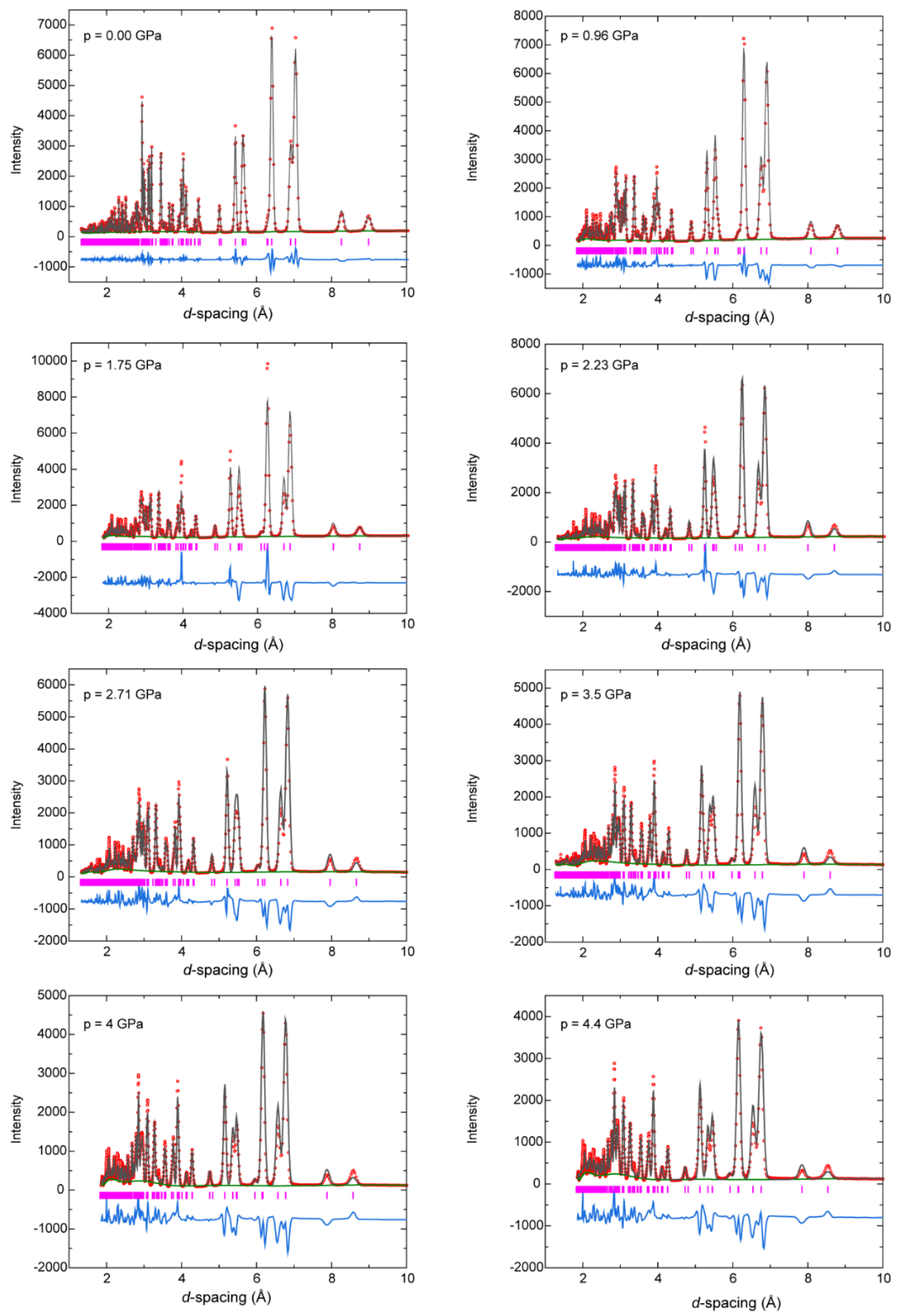

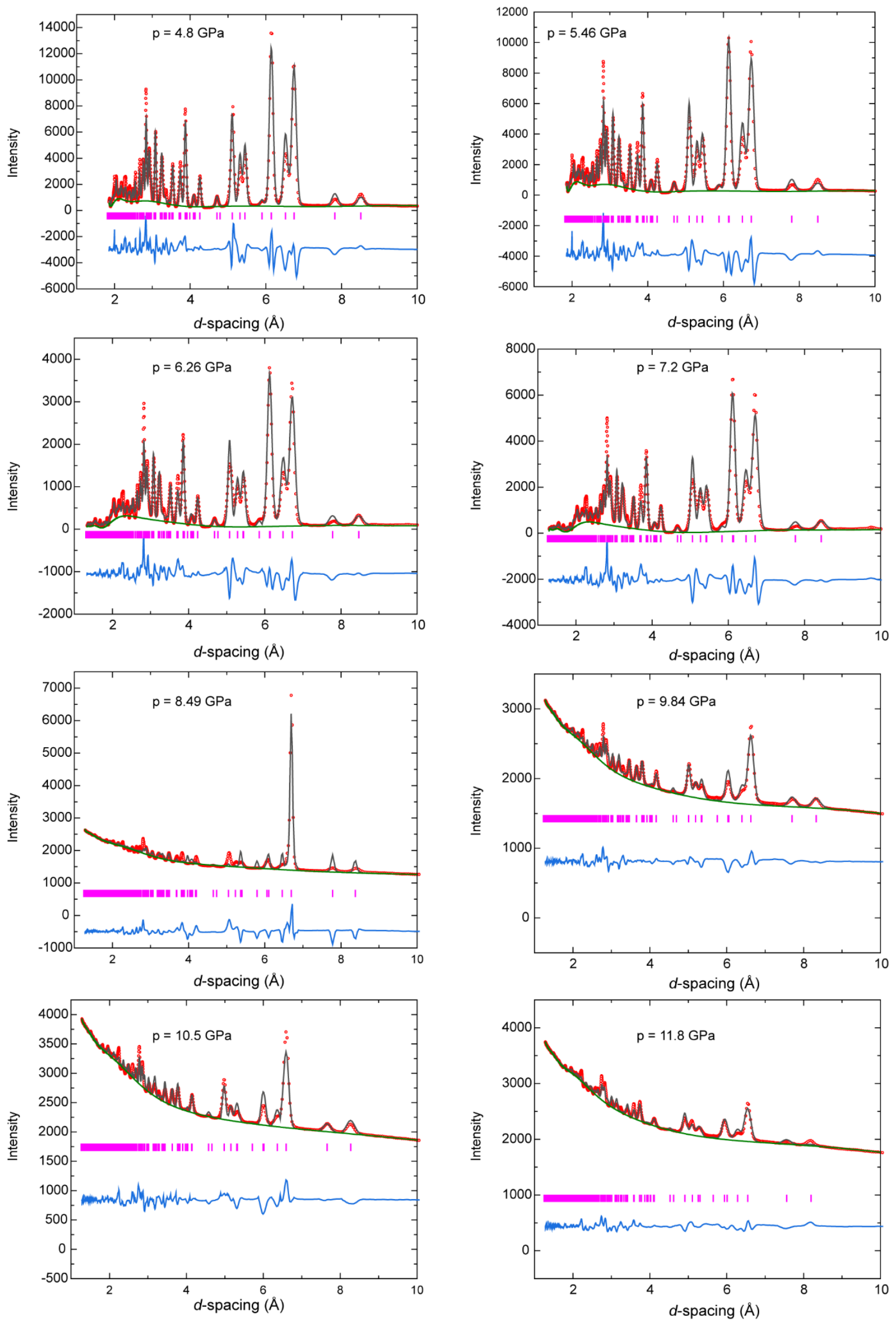
Figure S4 Comparison of calculated XRPD patterns of $\varepsilon$ - and $\gamma$-form of CL-20

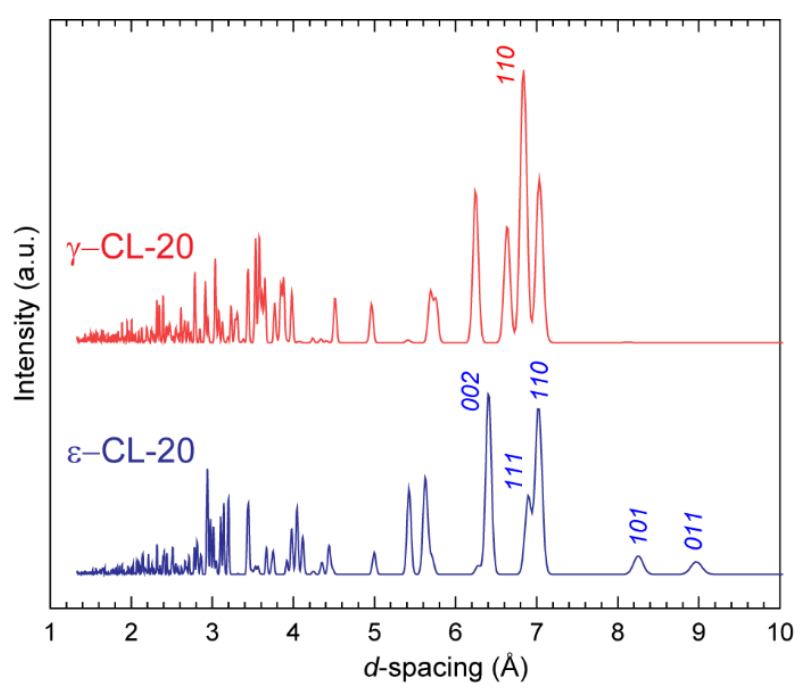


Figure S5 Rietveld refinement plots of Neutron powder diffraction patterns at elevated pressures
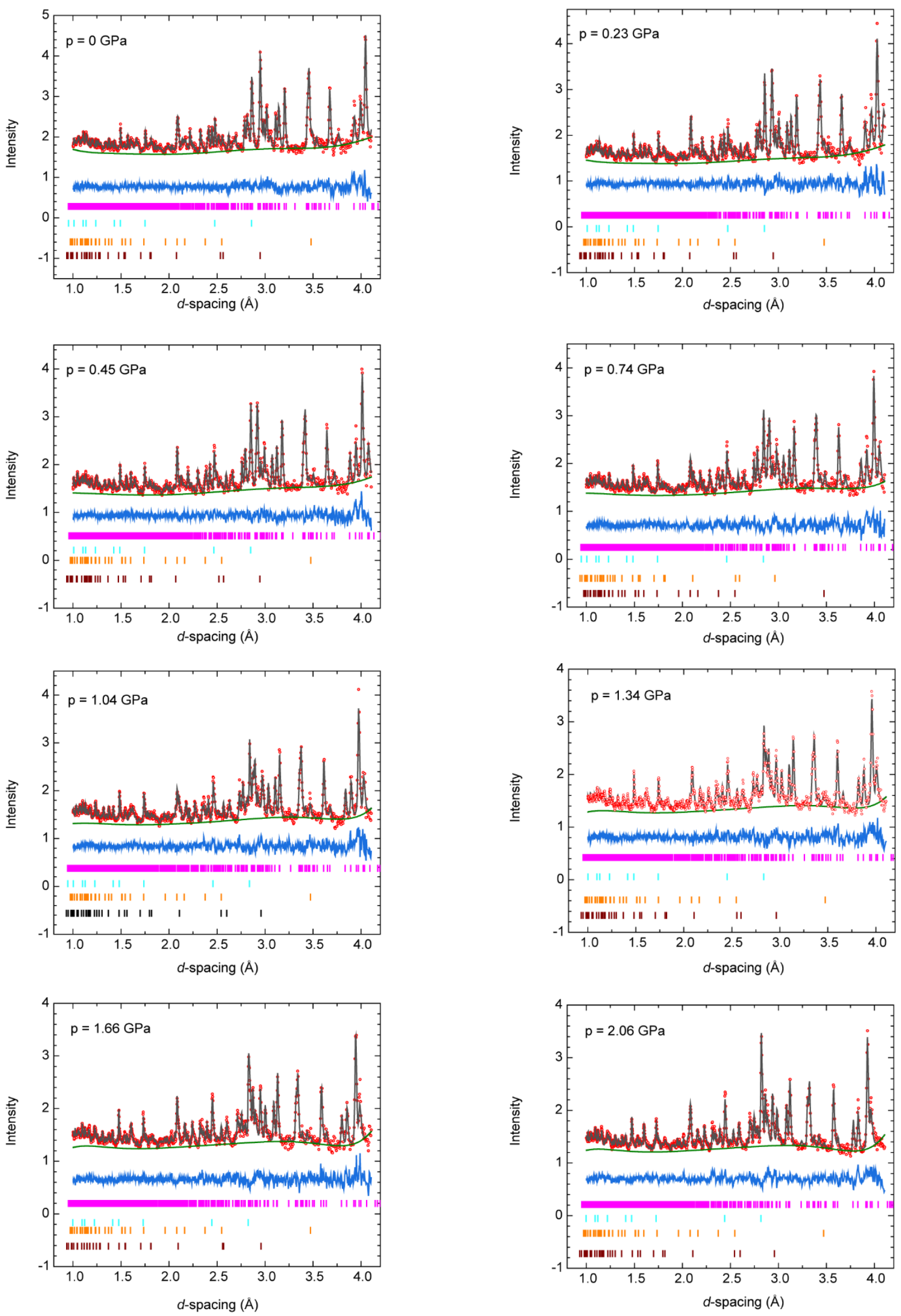

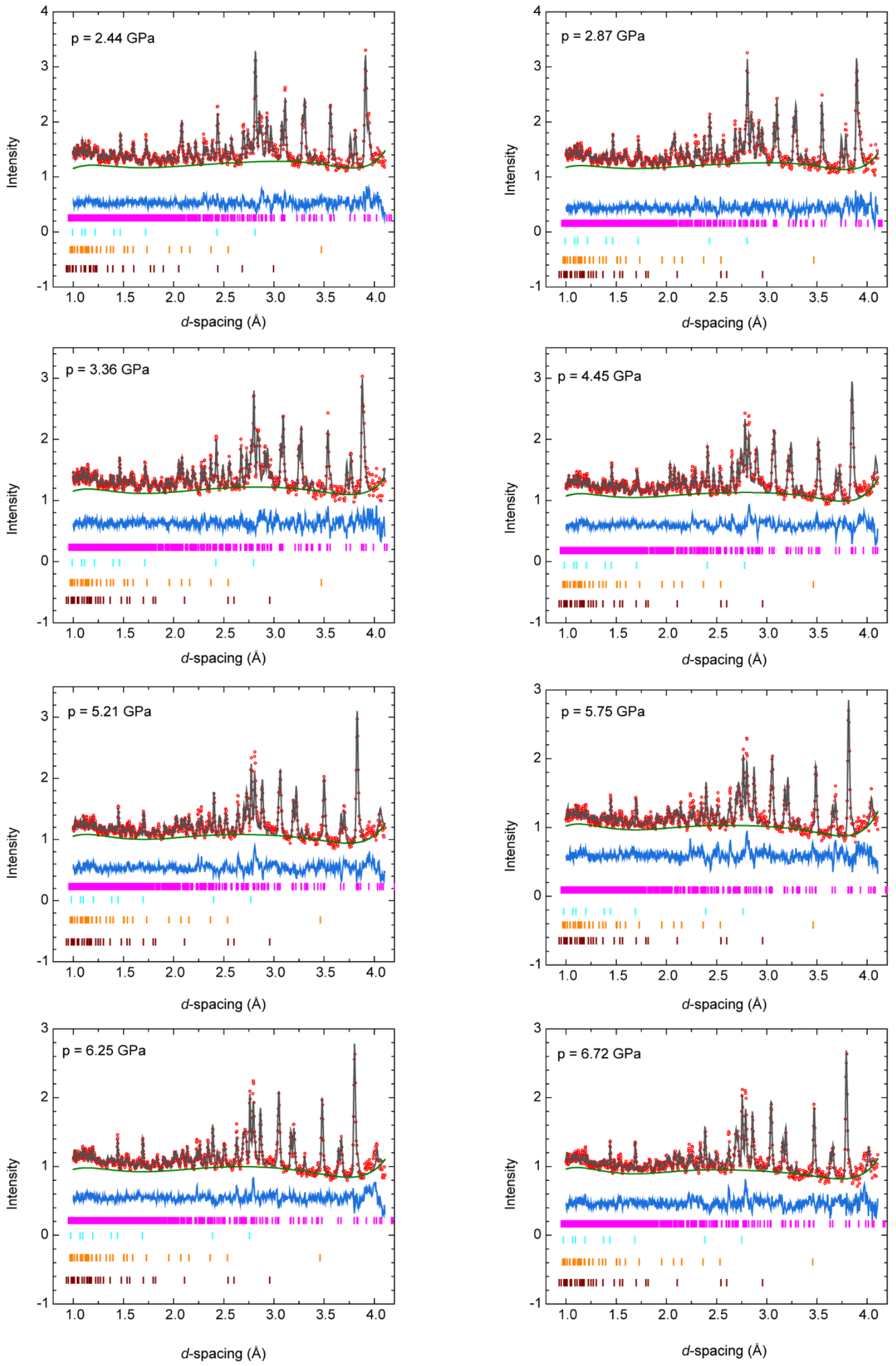


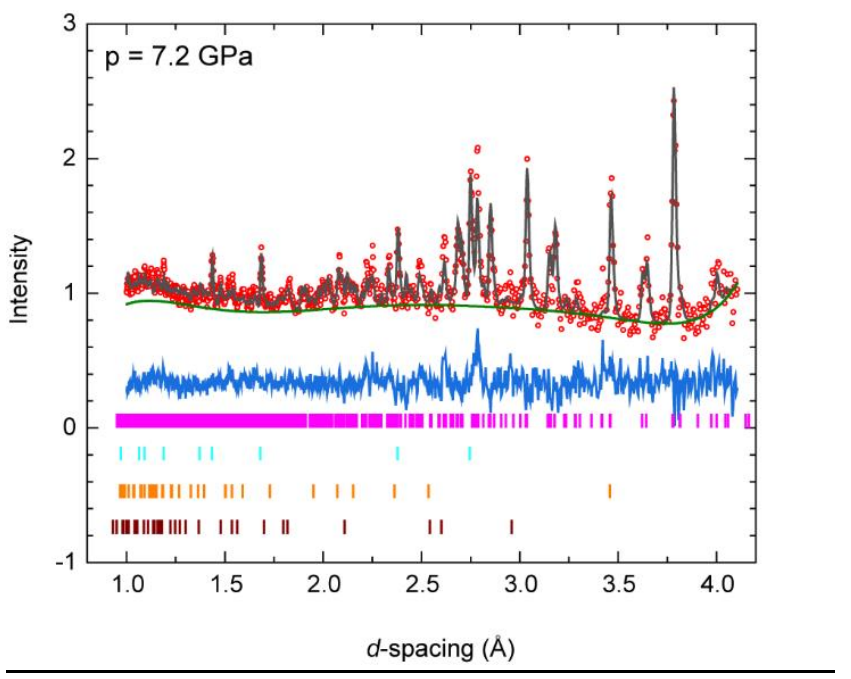


Table S6. Variation of unit cell parameters of $\varepsilon$-CL-20 with pressure obtained from Rietveld refinement of XRPD patterns. Values in parentheses are estimated standard deviations. Values in regular and italic font indicate input structures for refinement are taken from CCDC 117779 [Nielsen, A. T. et al. Tetrahedron 54, 11793-11812 (1998)] and DFT optimised structures (this work), respectively.

\begin{tabular}{|c|c|c|c|c|c|c|}
\hline Pressure. (GPa) & $\mathrm{a}(\AA)$ & $\mathrm{b}(\AA)$ & $c(\AA)$ & $\beta$ (deg) & $\mathrm{V}\left(\AA^{3}\right)$ & wRp \\
\hline \multirow[b]{2}{*}{0} & $8.8669(2)$ & $12.6023(3)$ & $13.3916(3)$ & $106.891(2)$ & $1431.87(4)$ & 0.0606 \\
\hline & $8.8669(3)$ & $12.6022(4)$ & 13.3913(3) & $106.890(2)$ & $1431.83(4)$ & 0.0669 \\
\hline \multirow{3}{*}{$0.9(2)$} & $8.6987(8)$ & $12.291(1)$ & $13.121(1)$ & $106.331(7)$ & $1346.3(1)$ & 0.119 \\
\hline & $8.6977(6)$ & $12.2913(8)$ & $13.1224(8)$ & $106.325(5)$ & $1346.3(1)$ & 0.0886 \\
\hline & $8.660(1)$ & $12.201(1)$ & $13.044(1)$ & $106.09(1)$ & $1324.2(2)$ & 0.1497 \\
\hline $1.7(2)$ & $8.6585(9)$ & $12.201(1)$ & $13.048(1)$ & $106.089(9)$ & $1324.4(2)$ & 0.1341 \\
\hline \multirow[t]{2}{*}{$2.2(2)$} & $8.637(1)$ & $12.149(1)$ & $12.999(1)$ & $105.964(9)$ & $1311.3(2)$ & 0.1675 \\
\hline & $8.6348(7)$ & $12.149(1)$ & $13.002(1)$ & $105.956(7)$ & $1311.4(1)$ & 0.1366 \\
\hline \multirow[t]{2}{*}{$2.7(2)$} & $8.603(1)$ & $12.070(2)$ & $12.927(1)$ & $105.74(1)$ & $1291.9(2)$ & 0.1696 \\
\hline & $8.6003(7)$ & $12.070(1)$ & $12.931(1)$ & $105.749(7)$ & $1291.9(1)$ & 0.1150 \\
\hline \multirow[t]{2}{*}{$3.5(2)$} & $8.556(1)$ & $11.959(2)$ & $12.829(2)$ & $105.39(1)$ & $1265.7(2)$ & 0.191 \\
\hline & $8.5525(8)$ & $11.962(1)$ & $12.833(1)$ & $105.393(8)$ & $1265.9(2)$ & 0.1393 \\
\hline \multirow[t]{2}{*}{$4.0(2)$} & $8.541(2)$ & $11.915(2)$ & $12.795(2)$ & $105.25(2)$ & $1256.3(3)$ & 0.2021 \\
\hline & $8.536(1)$ & $11.920(2)$ & $12.799(2)$ & $105.25(1)$ & $1256.4(2)$ & 0.1490 \\
\hline \multirow[t]{2}{*}{$4.4(2)$} & $8.518(2)$ & $11.848(3)$ & $12.742(2)$ & $105.04(2)$ & $1241.9(3)$ & 0.2086 \\
\hline & $8.515(1)$ & $11.845(2)$ & $12.745(2)$ & $105.00(1)$ & $1241.7(2)$ & 0.1349 \\
\hline \multirow[t]{2}{*}{$4.8(2)$} & $8.506(2)$ & $11.801(3)$ & $12.716(2)$ & $104.88(2)$ & $1233.7(3)$ & 0.2038 \\
\hline & $8.504(1)$ & $11.793(2)$ & $12.718(2)$ & $104.82(2)$ & $1233.0(2)$ & 0.1468 \\
\hline \multirow[t]{2}{*}{$5.46(2)$} & 8.49214 & $11.760(4)$ & $12.687(3)$ & $104.68(2)$ & $1225.6(4)$ & 0.2065 \\
\hline & $8.489(2)$ & $11.753(3)$ & $12.687(2)$ & $104.62(2)$ & $1224.7(3)$ & 0.1700 \\
\hline \multirow[t]{2}{*}{$6.26(2)$} & $8.477(2)$ & $11.713(4)$ & $12.658(2)$ & $104.52(2)$ & $1216.7(4)$ & 0.2006 \\
\hline & $8.474(2)$ & $11.702(3)$ & $12.659(2)$ & $104.48(2)$ & $1215.5(3)$ & 0.1694 \\
\hline \multirow[t]{2}{*}{$7.22(2)$} & $8.461(3)$ & $11.672(4)$ & $12.624(3)$ & $104.43(2)$ & $1207.3(4)$ & 0.2099 \\
\hline & $8.455(2)$ & $11.662(3)$ & $12.629(2)$ & $104.41(2)$ & $1206.2(3)$ & 0.1744 \\
\hline $8.49(2)$ & $8.486(3)$ & $11.591(9)$ & $12.527(7)$ & $104.85(4)$ & $1191.0(8)$ & 0.0345 \\
\hline $9.84(2)$ & $8.384(3)$ & $11.504(6)$ & $12.473(4)$ & $104.79(3)$ & $1163.2(5)$ & 0.0147 \\
\hline $10.5(5)$ & $8.346(4)$ & $11.430(7)$ & $12.396(5)$ & $104.78(4)$ & $1143.3(5)$ & 0.0175 \\
\hline $11.8(5)$ & $8.274(4)$ & $11.300(7)$ & $12.251(5)$ & $104.05(5)$ & $1111.3(7)$ & 0.0125 \\
\hline
\end{tabular}


Table S7. Variation of unit cell parameters of $\varepsilon$-CL-20 with pressure, obtained from Rietveld refinement of NPD patterns. Values in parentheses are estimated standard deviations. Values in regular and italic font indicate input structures for refinement are taken from CCDC 117779 [Nielsen, A. T. et al. Tetrahedron 54, 11793-11812 (1998)] and DFT optimised structures (this work), respectively.

\begin{tabular}{|c|c|c|c|c|c|c|}
\hline Pressure.(GPa) & $\mathrm{a}(\AA)$ & $\mathrm{b}(\AA)$ & $c(\AA)$ & $\beta(\operatorname{deg})$ & $\mathrm{V}\left(\AA^{3}\right)$ & $w R p$ \\
\hline \multirow{2}{*}{$0.00(1)$} & $8.8629(9)$ & $12.596(2)$ & $13.380(1)$ & $106.917(8)$ & $1429.0(2)$ & 0.0229 \\
\hline & $8.863(1)$ & $12.595(2)$ & $13.379(1)$ & $106.913(8)$ & $1428.9(2)$ & 0.0230 \\
\hline \multirow{2}{*}{$0.22(1)$} & $8.8138(9)$ & $12.510(2)$ & $13.311(2)$ & $106.742(8)$ & $1405.6(2)$ & 0.0253 \\
\hline & $8.814(1)$ & $12.510(2)$ & $12.311(2)$ & $106.738(8)$ & $1405.5(2)$ & 0.0251 \\
\hline \multirow{2}{*}{$0.43(1)$} & $8.7739(8)$ & $12.443(1)$ & $13.253(1)$ & $106.608(8)$ & $1386.5(2)$ & 0.0242 \\
\hline & $8.7736(8)$ & $12.442(1)$ & $13.252(1)$ & $106.604(8)$ & $1386.3(2)$ & 0.0238 \\
\hline \multirow{2}{*}{$0.71(1)$} & $8.7295(9)$ & $12.354(1)$ & $13.181(2)$ & $106.405(9)$ & $1363.6(2)$ & 0.0267 \\
\hline & $8.7291(8)$ & $12.354(1)$ & $13.181(1)$ & $106.407(8)$ & $1363.5(2)$ & 0.0240 \\
\hline \multirow{2}{*}{$1.01(1)$} & $8.6906(9)$ & $12.277(1)$ & $13.111(1)$ & $106.229(8)$ & $1343.1(2)$ & 0.0269 \\
\hline & $8.690(1)$ & $12.277(1)$ & $13.112(1)$ & $106.232(7)$ & $1343.0(2)$ & 0.0236 \\
\hline \multirow{2}{*}{$1.31(1)$} & $8.6571(9)$ & $12.205(1)$ & $13.049(1)$ & $106.081(9)$ & $1324.8(2)$ & 0.0263 \\
\hline & $8.6567(7)$ & $12.204(1)$ & $13.050(1)$ & $106.081(8)$ & $1324.7(2)$ & 0.0231 \\
\hline \multirow{2}{*}{$1.63(1)$} & $8.6238(9)$ & $12.139(2)$ & $12.992(2)$ & $105.903(9)$ & $1307.9(2)$ & 0.0282 \\
\hline & $8.6236(8)$ & $12.137(1)$ & $12.993(1)$ & $105.900(8)$ & $1307.9(2)$ & 0.0246 \\
\hline \multirow{2}{*}{$2.04(1)$} & $8.5912(8)$ & $12.063(1)$ & $12.928(1)$ & $105.713(8)$ & $1289.7(2)$ & 0.0261 \\
\hline & $8.5912(6)$ & $12.063(1)$ & $12.929(1)$ & $105.716(6)$ & $1289.8(1)$ & 0.0197 \\
\hline \multirow{2}{*}{$2.40(1)$} & $8.5607(9)$ & $11.994(2)$ & $12.866(2)$ & $105.527(9)$ & $1272.9(2)$ & 0.0294 \\
\hline & $8.5613(7)$ & $11.995(1)$ & $12.869(1)$ & $105.544(7)$ & $1273.2(1)$ & 0.0230 \\
\hline \multirow{2}{*}{$2.83(1)$} & $8.5322(9)$ & $11.925(2)$ & $12.808(2)$ & $105.37(1)$ & $1256.6(2)$ & 0.0315 \\
\hline & $8.5324(7)$ & $11.926(1)$ & $12.810(1)$ & $105.373(8)$ & $1256.8(2)$ & 0.0252 \\
\hline \multirow{2}{*}{$3.34(1)$} & $8.500(1)$ & $11.854(2)$ & $12.742(2)$ & $105.15(1)$ & $1239.3(2)$ & 0.0342 \\
\hline & $8.5016(8)$ & $11.855(1)$ & $12.743(1)$ & $105.157(9)$ & $1239.6(2)$ & 0.0286 \\
\hline \multirow{2}{*}{$4.44(2)$} & $8.448(1)$ & $11.724(2)$ & $12.621(2)$ & $104.80(1)$ & $1208.6(2)$ & 0.0314 \\
\hline & $8.4474(7)$ & $11.723(1)$ & $12.622(1)$ & $104.79(1)$ & $1208.5(1)$ & 0.0209 \\
\hline \multirow{2}{*}{$5.20(2)$} & $8.416(1)$ & $11.642(2)$ & $12.547(2)$ & $104.60(1)$ & $1189.6(2)$ & 0.0357 \\
\hline & $8.4145(7)$ & $11.640(1)$ & $12.547(1)$ & $104.59(1)$ & $1189.4(1)$ & 0.0236 \\
\hline \multirow{2}{*}{$5.75(2)$} & $8.394(1)$ & $11.592(2)$ & $12.501(2)$ & $104.49(2)$ & $1177.7(2)$ & 0.0409 \\
\hline & $8.3933(8)$ & $11.590(2)$ & $12.501(1)$ & $104.48(1)$ & $1177.4(2)$ & 0.0280 \\
\hline \multirow{2}{*}{$6.24(2)$} & $8.373(1)$ & $11.549(2)$ & $12.455(1)$ & $104.33(1)$ & $1167.0(2)$ & 0.0313 \\
\hline & $8.3735(7)$ & $11.547(1)$ & $12.456(1)$ & $104.33(1)$ & $1166.9(1)$ & 0.0228 \\
\hline \multirow{2}{*}{$6.70(2)$} & $8.360(1)$ & $11.509(2)$ & $12.422(2)$ & $104.25(2)$ & $1158.4(2)$ & 0.0412 \\
\hline & $8.3595(8)$ & $11.506(2)$ & $12.421(1)$ & $104.24(1)$ & $1158.1(2)$ & 0.0285 \\
\hline \multirow{2}{*}{$7.18(3)$} & $8.344(2)$ & $11.472(3)$ & $12.391(2)$ & $104.18(2)$ & $1149.9(3)$ & 0.0494 \\
\hline & $8.344(1)$ & $11.469(2)$ & $12.391(2)$ & $104.18(1)$ & $1149.7(2)$ & 0.0375 \\
\hline
\end{tabular}


Figure S6 Intermolecular distances (d1-d8) monitored in the PIXEL calculations. The distances quoted here are obtained from computational cif file at ambient pressure.
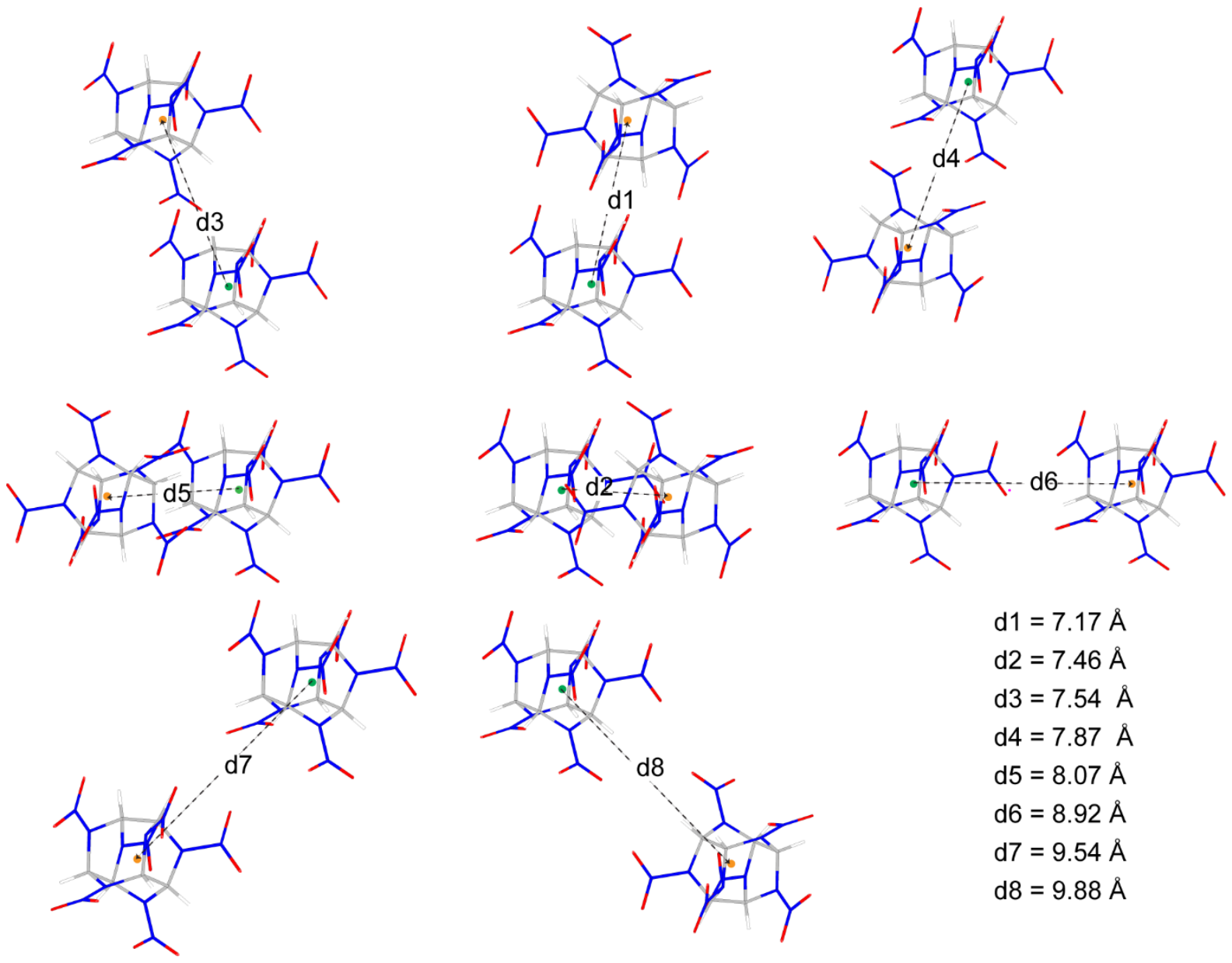

$$
\begin{aligned}
& \mathrm{d} 1=7.17 \AA \\
& \mathrm{d} 2=7.46 \AA \\
& \mathrm{d} 3=7.54 \AA \\
& \mathrm{d} 4=7.87 \AA \\
& \mathrm{d} 5=8.07 \AA \\
& \mathrm{d} 6=8.92 \AA \\
& \mathrm{d} 7=9.54 \AA \\
& \mathrm{d} 8=9.88 \AA
\end{aligned}
$$

